\section{región
sociedad}

Ruiz Luna, Arturo; Madrid Vera, Juan

Análisis comparativo de tres sistemas de pesca artesanal

Región y Sociedad, vol. VIII, núm. 13, enero-diciembre, 1997

El Colegio de Sonora

Hermosillo, México

Available in: http://www.redalyc.org/articulo.oa?id=10201303

How to cite

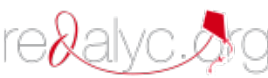

Complete issue

- More information about this article

Journal's homepage in redalyc.org

Scientific Information System Network of Scientific Journals from Latin America, the Caribbean, Spain and Portugal Non-profit academic project, developed under the open access initiative 


\title{
Análisis comparativo de tres sistemas de pesca artesanal
}

\author{
Arturo Ruiz Luna* \\ Juan Madrid Vera*
}

Resumen: Se examinan dos sistemas de pesca artesanal del Pacífico mexicano y uno del Mediterráneo español; $\$$ analizan aspectos de producción,diversidad biológica y manejo, con el objeto de conocer en qué medida son determinantes para el desarrollo de esta actividad. Pese a diferencias geográficas y culturales, se observaron similitudes entre los sistemas de producción, no así en su organización. En los sistemas mexicanos, el capital destinado al mantenimiento y mejoramiento de instalaciones y equipo es limitado, lo que se refleja en el nivel de vida de los operarios, a diferencia del sistema español. La infraestructura, así como la presencia de centros de capacitación no resulta ron determinantes. Las diferencias se atribuyen a los hábitos y políticas para la diversificación del consumo y a la inversión de capital, así como a una particular regulación del esfuerzo pesquero (número de embarcaciones/ día) y de la comercia lización de la producción en la pesquería española, cuya viabilidad se discute para las pesquerías mexicanas.

Abstract: Two craftsmanship-type fishing systems of the Mexican Pacific and one of the Spanish Mediterranean are analyzed, as regards production aspects, biological diversity and management, order to establish the degree in which they are deter-

* Centro de Investigación en Alimentación y Desarrollo. Unidad Mazatlán Se le puede enviar correspondencia a CIAD, Unidad Mazatlán,Apdo. postal 711. Ma zatlán, Sinaloa. Tels: (69) 88-01-59 y 88-01-57, c. electrónico arluna@ servidor.unam.mx.

** Centro Regional de Investigación Pesquera Manzanillo. INP. Se le puede enviar correspondencia a PlayaVentanas, s/ n.Manzanillo, Colima,México. 
mining for the development of this activity. Despite geograph-ical and cultural differences, similarities were observed between the production systems although there were important differences regarding their organization. In the Mexican Systems, capital for maintenance and improvement of facilities and equipment is limited, being reflected in the level of life of the operators, as opposed to the Spanish system.The infrastructure, as well as the presence of training centers were not determining. Differences are attributable to customs and policies for diversifying consumption and to capital investments, as well as to a particular regulation of fishing efforts (number of vessels per day) and production marketing in Spanish fisheries, whose feasibility is discussed for Mexican fisheries.

\section{Introducción}

La pesca artesanal, ribereña o de pequeña escala, se asocia con estratos socioeconómicos bajos y aún cercanos al nivel de subsistencia en los países en desarrollo. Actualmente, ocupa a más de $10,000,000$ pescadores en el mundo y produce cerca del $50 \%$ de la producción pesquera destinada al consumo humano directo.

Esta actividad fue vista como un paso previo a la pesca industrial, con el presumible mejoramiento del nivel de vida del pescador. El constante aumento en los costos de producción y el deterioro progresivo de los recursos han modificado visiblemente esta idea, por lo que, actualmente, se busca fortalecer la pesca costera y la acuicultura. 1

La diversidad de técnicas de captura en estos sistemas dificulta su caracterización, aunque es distintiva la escasa mecanización, el uso de reducidos capitales y mayor empleo de mano de obra. La 
rentabilidad es variable de una pesquería a otra, lo que indica que no es el tipo de pesca lo que impide su desarrollo, sino que hay otras determinantes, posiblemente de carácter social, cultural y económico que deben considerarse en la investigación como un prerrequisito para conseguir un adecuado plan de manejo de estos sistemas. ${ }^{2}$

En este trabajo, se compara una pesquería artesanal rentable, ubicada en el Mediterráneo español, con dos sistemas mexicanos con baja rentabilidad, a fin de determinar en que medida la naturaleza de las capturas, la variación de los métodos y técnicas de pesca y el manejo de la producción, tienen efecto sobre la viabilidad económica de la pesca artesanal en su respectiva área geográ fica. La elección de los sitios obedece a la experiencia previa que sobre estos tienen los autores y que dio lugar al planteamiento central del problema.

\section{Ubicación y características de los sistemas}

Por su ubicación geográfica y evolución como modelos de orga nización social, los sistemas seleccionados difícilmente podrían ser comparados, sin embargo, se posibilita al aplicar criterios biológicos y de valoración económica. Cabe señalar que se tiene un conocimiento previo de estas pesquerías, por lo que se facilitó la selección de los factores a evaluar. ${ }^{3}$

Con respecto a las localidades del Pacífico mexicano, Mazatlán, Sinaloa y Bahía Bufadero, Michoacán, (figura 1), existen similitudes en términos ambientales, aunque demográficamente y a nivel de infraestructura básica existen diferencias notables. Mazatlán es mayor en extensión y población, además de que cuenta con ser-

2 Max Agüero (1992),pp. 1-27

3 Arturo Ruiz Luna (1983), p. 137; (1989), p. 335; (1991),pp. 89-98; Martha Elena Macías Romo y Asminda Felicidad Mota Pineda (1990), pp. 154; Juan Madrid Vera, (1990); Mario Soria Flores (1992); Juan Madrid Vera, et al. 


\section{Figura 1}

U bicación de las zonas de estudio

en sus respectivas entidades federativas y su capital. Mazatlán, Sinaloa y Bahía Bufadero, Michoacán

vicios primarios (salud,educación, comunicaciones, agua potable, etcétera) disponibles para la mayor parte de la población.

Pese a las diferencias socioeconómicas, la actividad pesquera artesanal es similar en ambas regiones, con la embarcación de fibra de vidrio y motor fuera de borda, además de los artes de pesca como la unidad básica de pesca, que opera dentro de un radio aproximado de $15 \mathrm{~km}$ sobre la costa, principalmente en zonas someras, más activamente en áreas rocosas, dentro de la plataforma continental, muy estrecha en esta franja del Pacífico. 
Ambas regiones presentan dos estaciones bien definidas; una época de secas (noviembre-junio), donde se obtiene el máximo de producción y otra de lluvias (julio-octubre), que afecta la actividad pesquera ya que son frecuentes las tormentas tropicales con vientos de alta intensidad que impiden la navegación. Cabe desta car que en la época de lluvias inicia la temporada de captura de camarón (Penaeus spp.), recurso de gran importancia, particularmente para Sinaloa. Sin embargo, dadas las peculiaridades de este recurso, se considera como un objeto de estudio aparte.

Bahía Bufadero es una localidad rural, que se ubica aproximada mente a 70 km al norte del puerto de Lázaro Cárdenas, principal centro urbano en la costa michoacana. Actualmente, cuenta con más de 5,000 habitantes y ha mostrado una tasa de crecimiento elevada a partir de los 80 's, cuando su población era aproximada mente de 2,000 habitantes. 4 Pese a ello, el número de pescadores se ha mantenido cercano a 50-70 operarios de acuerdo a la época del año y ha mostrado un crecimiento en otras áreas de la economía, tales como agricultura, comercio y servicios turísticos.

En términos generales, la población económicamente activa (PEA) se sitúa en $\mathbf{4 0 \%}$, aunque varía temporalmente debido a procesos de migración, principalmente del sector masculino, que se desplaza hacia los Estados Unidos en busca de oportunidades de trabajo. No se cuenta con información al respecto, pero por observaciones en la zona, se estima que un elevado porcentaje de los emigrantes vuelven debido a la carencia de permisos para laborar en los E.U.A., o bien para permanecer una temporada con su familia. Asimismo, una parte de la población infantil y del sexo femenino está subempleada en actividades de comercio informal, principalmente relacionadas con el turismo (venta de alimentos en establecimientos informales y por mercadeo ambulante).

Las principales actividades son el comercio, la agricultura, la pesca, el turismo y otros servicios. Algunas tareas se practican simultáneamente, en especial la agricultura y la pesca, de hecho, los pescadores tienen extracción agrícola. Este origen se infiere con- 
siderando que la fundación de Bahía Bufadero es a partir de los años 50 y que algunos de los pescadores y habitantes con otras actividades proceden de sitios alejados de la costa.

Por el contrario, Mazatlán es un puerto tradicionalmente pes quero y turístico, con más de 300,000 habitantes, de los que alrededor del $32 \%$ forman la PEA. De éstos, el $12.2 \%$ corresponde al sector primario, aunque no se cuenta con la información disponible respecto al personal que se dedica a la pesca. 5

En este puerto existen instituciones educativas y de investiga ción que forman personal desde niveles técnicos hasta posgrado en diversas disciplinas asociadas a la investigación pesquera, a diferencia de Bahía Bufadero, donde el nivel máximo que se imparte es el de Secundaria Técnica.

Por lo que respecta a la pesquería española, ésta se establece en Sant Carles de la Ràpita, en Cataluña, formando parte del sistema estuarino del delta del Ebro (figura 2). En esta región, las estaciones del año están bien definidas, con fuertes vientos durante la primavera que limitan, pero no impiden la captura. Asimismo, durante el invierno hay reducción de la producción debido al descenso de la temperatura del agua, cercano a $10^{\circ} \mathrm{C}$ en el mes de febrero. ${ }^{6}$

La pesca artesanal se realiza en el interior e inmediaciones de la bahía Els Alfacs, que tiene una extensión aproximada de $50 \mathrm{~km}{ }^{2}$, con profundidad media de $4 \mathrm{~m}$ y comunica al norte con sistemas lagunares y al suroeste con el Mediterráneo, actuando como zona de tránsito y crianza para varias especies acuáticas de importancia comercial. ${ }^{7}$

El origen de esta pesquería se remonta al siglo XI y dependen de ella casi el $10 \%$ de los habitantes de esta población, ${ }^{8}$ cuyo nú123.

6 Max Delgado (1986), p. 261.

7 Arturo Ruiz Luna (1989), p. 335.

8 R. L.Millán (1986), p. 212. 


\section{Figura 2}

Localización de la Bahía Els Alfacs, España

mero actual se aproxima a 10,000. Esta población cuenta con un puerto pesquero y otro de usos múltiples, principalmente, para fines comerciales y recreativos. Asimismo, existen astilleros propios e instalaciones para la conservación, comercialización y distribución de la captura; realizándose la venta se realiza a través del sistema de Lonja (subasta) y cuenta con servicio de informática por medio del que se registra la producción diaria. 
Sant Carles de la Ràpita ocupa el tercer lugar en facturación de productos de la pesca en Cataluña, el mayor aporte corresponde a la flota de arrastre, por lo que las principales especies son demersales y semi-pelágicas (lenguados, clupeidos, merlúcidos, etcétera) aunque a diferencia de otros puertos, su producción no se basa en la captura de sardina, que para los otros representa más del $60 \%$. La captura obtenida en la bahía es menor al $10 \%$ del total facturado en ese puerto, que alcanza aproximadamente las 6,000 toneladas por año. ${ }^{9}$

Asimismo, es un centro turístico de mediana importancia y su principal atractivo reside en la infraestructura portuaria para la pesca recreativa y la navegación, además de contar con un considerable número de establecimientos gastronómicos especializados en pescados y mariscos.

\section{Producción y régimen de pesca}

Michoacán es la quinta entidad federativa del litoral del Pacífico en producción pesquera, sin embargo, más del $85 \%$ de las capturas corresponden a producción continental y la mayor parte de la pesca de origen marino es producida por la pesca artesanal, aproximadamente 2,000 toneladas por año. ${ }^{10}$ En Bahía Bufadero la captura es multiespecífica y diversificada, rasgo común en los sistemas estudiados y que en general, se comparte con la pesca artesanal. No hay estimaciones confiables de la producción anual, y se calcula entre 250 y 300 тм. ${ }^{11}$

Para el periodo en el cual se evaluó la composición y diversidad de la pesca en esta zona (1987-92), la media de captura por barca se situó dentro de un intervalo de 24 a $90 \mathrm{~kg}$, dependiendo de la temporada, horas de pesca y operarios por barca. 12

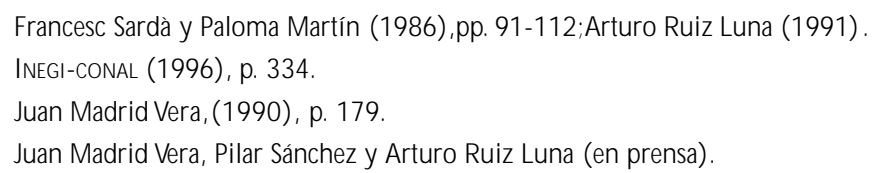


El número de pescadores en activo depende de la disponibilidad de barcas, normalmente insuficiente. La SEPESCA reportó más de 3000 embarcaciones y un número similar de pescadores dedica dos a la pesca artesanal, sin embargo, no discrimina entre pesca marina y continental. En términos generales, la unidad básica de pesca en Bahía Bufadero se compone de 2-3 pescadores por lancha de fibra de vidrio de $6 \mathrm{~m}$ de eslora y motor fuera de borda de 25 a 65 HP. ${ }^{13}$ La duración de la jornada es de aproximadamente ocho horas, y puede combinar operaciones tales como el calado de redes 0 cimbras (palangres) y la pesca con anzuelo, o bien dedicarse exclusivamente a una modalidad de pesca (anzuelo, buceo libre, pesca con red, etcétera).

Por el sistema de remuneración predominante en la región, con pago inmediato a la venta del producto, no se destina parte de la ganancia al mantenimiento del equipo; ya han fracasado diversos intentos de creación de un fondo de esta naturaleza por problemas de corrupción, malos manejos y desconfianza de los pescadores (observación personal) .Actualmente, se continúa con esa práctica de pago inmediato.

Por tal razón en la mayoría de los casos, son los pescadores quienes realizan las reparaciones y dan mantenimiento al equipo, aunque no siempre cuentan con la preparación y refacciones adecuadas, genera desempleo temporal, arrendamiento de equipo a permisionarios y pesca irracional de especies más susceptibles de sobreexplotación (lapa, caracoles, ostión, langosta) para las que se requiere un mínimo de equipo (visor, aletas y gancho o barra de acero).

En Mazatlán la situación es similar pese a la existencia de mayor infraestructura.La principal actividad pesquera se centra en la captura del camarón (Penaeus spp.), para lo cual se cuenta con una flota de arrastre de más de 500 embarcaciones. 0 tros recursos importantes desembarcados en este puerto son el atún y la sardina (Opisthonema spp.). En conjunto, se obtiene un volumen aproxima do de 120,000 t/ año en Sinaloa, de las que cerca del $70 \%$ son re- 
portadas en Mazatlán. ${ }^{14}$ Este puerto es el principal productor de camarón en el país y, actualmente, se sitúa como uno de los primeros centros de desembarco de atún.

Como en el caso anterior, la captura diaria obtenida por la flota artesanal, presenta fluctuaciones tanto en diversidad como en volumen, y se ubica dentro de un intervalo de 5 a $20 \mathrm{~kg}$ por persona. ${ }^{15}$ Asimismo, la disponibilidad de barcas es siempre menor a la demanda debido al mal estado de los motores y a la escasez de recursos destinados para su mantenimiento y reparación, y es frecuente que unidades en buen estado estén en proceso de deterioro al carecerse de equipo para su uso.

Los principales artes de pesca que se emplean son, como en Michoacán,líneas y anzuelos y redes que varían en tipo y tamaño del paño y la abertura de malla, de acuerdo al producto que se espera capturar. A diferencia de Bahía Bufadero, existe especialización en algunos grupos de pescadores para la captura de langosta, ostión y pesca con arpón.

En la bahía de Els Alfacs se tiene registrada una captura media anual de $325 \mathrm{TM}$, con un promedio diario de $23.4 \mathrm{~kg}$ por barca es, asimismo, una pesquería multiespecífica y con diversificación en las técnicas de captura. 16

Contrariamente a lo que ocurre con los sistemas mexicanos, donde las condiciones ambientales y carencia de equipo restan continuidad a la actividad, esta pesquería se caracteriza por mantener activa de manera continua a la flota pesquera, contabilizando una media de 290 días en activo por año, con un promedio de 48 embarcaciones diarias que reportaron captura. La pesca se realiza de lunes a sábado, con una particular administración del esfuerzo pesquero que permite mantener adecuados precios de venta.

La estrategia consiste en reducir el número de unidades en operación durante los días lunes, miércoles y sábado; se incrementa 
notablemente en martes y, especialmente, en jueves y viernes, días previos a una elevación en la demanda (figura 3). Este esquema sigue sin cambios durante todo el año, con variaciones proporcionales en la temporada de mayor o menor producción. 17

Las pesquerías mexicanas, por su parte, aumentan el esfuerzo en la época de secas, donde obtienen capturas abundantes que llegan a saturar los mercados locales con el consiguiente descenso en el precio de venta en playa, con excepción de la época de cuaresma y fin de año, situación que beneficia a acaparadores y mayoristas con mayor capacidad para almacenar y distribuir la producción sin necesidad de abaratarla.

\section{Métodos de pesca y características de la captura}

Los principales artes de pesca empleados en las pesquerías mexicanas son, la línea de nylon con anzuelos de varios tipos y tama ños; palangres y redes de monofilamento, aunque estas últimas se utilizan principalmente para la captura de langosta, y se obtiene incidentalmente especies asociadas a zonas de arrecife. Las embarcaciones están construidas en fibra de vidrio, con eslora de $6 \mathrm{~m}$ y capacidad bruta de 1.5 T. En Mazatlán, existe además un importante número de barcas de madera con motor interno y capacidad de $0.5 \mathrm{~T}$.

Las especies capturadas son aquellas que ocupan los niveles tróficos superiores, tales como tiburones y perciformes en general, especialmente de las familias Lutjanidae, Serranidae, Carangidae y Scombridae (pargos, cabrillas, jureles y sierras, respectivamente), sobre las que se concentra la actividad mayor. La producción de langosta y ostión de roca también ocupa un lugar preponderante en estos sistemas,y se efectúa la extracción de este último por medio de inmersión a pulmón.

En la pesquería catalana existe una rotación estacional de los artes de pesca, asociados a determinadas especies con importancia 
REGIÓN Y SOCIEDAD / VOL. VIII / NO. 13-14. 1997

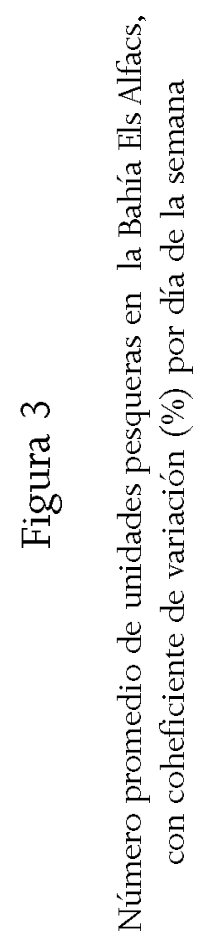


comercial. Durante la temporada verano-otoño, predomina el tras mallo, arte que consta de dos paños externos con una abertura de malla de 5" o mayor y un paño intermedio con aberturas de menor tamaño. Durante el resto del año, además de utilizar otros artes de enmalle, se usa principalmente el palangre con distintos tamaños de anzuelo. Pese a ello, a lo largo del año se emplean distintos artes que actúan sobre los diferentes ambientes presentes en la bahía.

La flota de artes menores de Els Alfacs tiene aproximadamente 200 embarcaciones con capacidad de 1 a 10 toneladas de registro bruto y con motor interno. La mayoría de las embarcaciones muestran especialización hacia recursos asociados con determinado arte de pesca, aunque es frecuente que utilicen de forma complementaria dos o más artes, incidiendo sobre una fracción más amplia de los recursos pesqueros dentro de la bahía.

Respecto a las capturas, se reconoce que en las regiones tropica les la diversidad de las especies es mayor en comparación con las templadas y con respecto a la fauna marina, es más evidente en la zona costera. Sin embargo, al evaluarse la diversidad de acuerdo a la teoría de la información, 18 donde cada individuo contiene elementos de información codificables en sistema binario (bits), no se observan diferencias substanciales en las capturas de los tres sistemas pese a que en las pesquerías mexicanas el número de especies capturadas es mucho mayor. Esto implica que a pesar del número de especies capturadas, la proporción de éstas que mayor volumen aporta a la pesquería es proporcional al número total de especies.

Lo anterior es producto de la menor diversificación de los métodos de pesca en los sistemas mexicanos, así como de las características de los ambientes, con una bahía semicerrada en Els Alfacs donde se efectúa una extracción intensiva, y bahías abiertas en Mazatán y Bahía Bufadero, con caladeros específicos conocidos.

A partir de las capturas obtenidas en épocas similares para Ma zatlán (1984), Bahía Bufadero (1982-83, 1987-89) y Els Alfacs 
(1982-84) se presenta un resumen en el cuadro 1. Los resultados pueden interpretarse en función del elevado número de especies que se captura incidentalmente en los sistemas mexicanos y de la diversificación en los métodos de pesca en Els Alfacs.

Es importante señalar la menor variabilidad $(S)$ de los valores de diversidad de la captura en Els Alfacs, que implica una constancia tanto en número como en la proporción de especies capturadas por esta pesquería.

\section{Comercialización}

En la localidad michoacana, la venta suele realizarse en la playa, directamente al consumidor y a restaurantes playeros, aunque es frecuente que los productos de mayor calidad comercial, tales como huachinangos y pargos (Lutjanidae), langostas y otros se hayan negociado de antemano con pequeños mayoristas que otorgan créditos para avituallamiento, proveen de hielo o simplemente se encargan del acopio y transportación del producto a cambio de que se les garantice la venta del total de la producción, en ocasiones con precios ventajosos.

En Mazatlán se da una situación similar, con varios puntos de venta directa en playa ( Playa norte, Embarcadero a la Isla de la Pie dra) y a comerciantes con locales establecidos para la venta de productos pesqueros. Normalmente, a éstos últimos se destina el producto de mayor calidad,mismo que no está sujeto a ningún tipo de manejo con excepción del eviscerado de los peces, luego de lo cual se enhiela para su transporte a los centros de acopio y distribución.

En el caso de Bahía Bufadero este tipo de productos se traslada a distancias considerables debido a que esta localidad se encuentra alejada de centros urbanos importantes, y es Lázaro Cárdenas, 70 km al sur, el punto más cercano. En Mazatlán, casi toda la producción de la pesca artesanal se consume localmente, y se desvía la de mayor calidad a prestadores de servicios turísticos. ${ }^{19}$ 


\section{Cuadro 1}

Características de las capturas

en tres sistemas de pesca artesanal

\begin{tabular}{|l|c|c|c|}
\hline Localidad & $\begin{array}{c}\text { Mazattán, Sin. } \\
\text { México }\end{array}$ & $\begin{array}{c}\text { B. Bufadero, Mich. } \\
\text { México }\end{array}$ & $\begin{array}{c}\text { Els Alfacs } \\
\text { España }\end{array}$ \\
\hline $\begin{array}{l}\text { No. de especies } \\
\text { reportadas }\end{array}$ & 103 & 188 & 41 \\
\hline $\begin{array}{l}\text { Media mensual } \\
\text { del índice de } \\
\text { diversidad de } \\
\text { ShannonWeaver }\end{array}$ & $\begin{array}{c}3.61 \\
\text { bits./ ind. }\end{array}$ & $\begin{array}{c}3.54 \\
\text { bits./ ind. }\end{array}$ & $\begin{array}{c}3.20 \\
\text { bits./ Kg. }\end{array}$ \\
\hline $\begin{array}{l}\text { Varianza (S2) } \\
\text { mensual del } \\
\text { índice de } \\
\text { diversidad }\end{array}$ & 0.17 & 0.21 & 0.10 \\
\hline $\begin{array}{l}\text { Aportación de } \\
\text { las 10 principales } \\
\text { especies a la } \\
\text { captura total }\end{array}$ & $74.1 \%$ & $68.03 \%$ & $82.97 \%$ \\
\hline
\end{tabular}

El precio promedio de venta es aproximadamente el equivalente a 3 dólares americanos por $\mathbf{k g}$ de producto de primera calidad, por lo que considerando una captura de $15 \mathrm{~kg}$ por unidad con tres operarios, se obtendrían alrededor de 15 dólares diarios por pescador, lo que equivaldría a 5-6 veces el salario mínimo actual. Esta estimación deberá ponderarse, al descontar una proporción de 20-30\% para gastos de operación (combustible y aceite) y considerarse que no existe garantía ni en la producción ni en la venta, lo que sitúa este sector productivo como uno de los más empo- 
brecidos del país. Este problema esta aparejado al grado de desa rrollo del país, ya que en países en desarrollo no hay mercado para los recursos de acceso abierto y por lo tanto no existe precio y en todo caso el precio asignado refleja el costo de oportunidad de la mano de obra. 20

En Els Alfacs existe un estricto control en la venta del producto, misma que se inicia diariamente a las 16:00 hrs en subasta pública que garantiza los mejores precios a los primeros lotes en venta. Un alto porcentaje de la producción se destina al consumo local, dado que Sant Carles de la Ràpita se considera un sitio de mucho atractivo para el consumo de pescados y mariscos de alta calidad, aunque también abastece a los mercados regionales, incluyendo Barcelona.

El hábito de consumo de pescados y mariscos frescos prevalece sobre cualquier otro tipo de procesamiento, por lo que sólo se recurre al enhielado y refrigeración antes de su venta.Asimismo, debe tomarse en cuenta que el consumo en esta región es bastante considerable y diversificado por lo que la mayoría de los productos que se capturan tienen salida comercial. ${ }^{21}$

En Cataluña, se tiene un precio de venta promedio para productos de la pesca de aproximadamente 1.6 dólares por $\mathrm{kg}$, aunque este valor incluye a especies de pesca masiva, mucho más baratas, tales como la sardina, pescadilla, diversos pelágicos y otras especies capturadas tanto por cerco como por el sistema de arrastre, por lo que atendiendo a la calidad de los productos obtenidos en Els Alfacs cabría esperarse que por lo menos debe alcanzarse un valor similar al de los sistemas mexicanos. En particular, debe considerarse que existen especies como la dorada (Sparus aurata) y salmonetes (M ullus Spp.) cuyos precios en el mercado son elevados, sobre todo dependiendo de la temporada y su abundancia en el medio.

Aunado a lo anterior, la regularidad de la actividad y el menor consumo de combustible, al operar básicamente dentro de la bahía, permiten estimar una ganancia neta de aproximadamente $\mathbf{4 0}$ 
dólares diarios por embarcación con uno o dos operarios, asumiendo un promedio de captura de 20-23 $\mathrm{kg}$ por barca.

\section{Discusión}

Considerando los tres casos, existen semejanzas en cuanto a los métodos y volúmenes de producción de los sistemas estudiados, así como en términos de la ganancia neta. Sin embargo, pese a que hay mayor variedad de la captura en las costas mexicanas, hay diferencias notorias en el nivel de vida, superior en los pescadores españoles. Una medida de este bienestar es la infraestructura con que cuenta el sector a nivel portuario y urbano, la garantía de servicios médicos y sanitarios adecuados, así como planes de retiro y seguridad social de las cuales se carece total o parcialmente en los sistemas mexicanos que se analizaron. Aunque esto es un refle jo de las economías de los países considerados, existen otros motivos que se considera conveniente resaltar con relación a la pesca en México.

En primer término, el consumo de productos de la pesca se encuentra escasamente arraigado en México y con excepción de las poblaciones costeras, es poco diversificado, lo que propicia eleva dos precios de las especies de mayor consumo. Cifras oficiales indican que aproximadamente el $\mathbf{5 0} \%$ de la captura nacional procede de los recursos masivos ( camarón, ctún, barrilete, sardinas, anchoveta) destinados a la exportación o la industria. Incluyendo el $15 \%$ que se reporta como otros y captura sin registro oficial, la producción que se destina a consumo humano directo en México es de aproximadamente $7.9 \mathrm{~kg}$ per capita. 22

Parte de este problema es la carencia de adecuados centros de acopio, distribución y abasto de productos de la pesca en zonas estratégicas. En distintas regiones del país, han surgido comunidades pesqueras aisladas de cualquier centro urbano por lo que, de no 
ser un producto destinado a la exportación (camarón, langosta, abulón) existen carencias infraestructurales básicas.

En general, la política pesquera mexicana ha favorecido eventualmente la pesca artesanal con la concesión de permisos, equipamiento y créditos, pero no con asesoría. Por ello, se desconocen los medios adecuados para hacer llegar su producción a un público más vasto que el local,situación que beneficia a un peque ño sector dedicado a la comercialización y no al pescador artesa nal que ofrece un producto carente de valor agregada. Esto es más evidente en Bahía Bufadero que en Mazatlán, donde se llega a filetear parte de la producción a fin de incrementar su valor. En Els Alfacs el producto también se ofrece fresco, pero el proceso de acopio y comercialización permite mayor transparencia en las transacciones y garantiza un precio de venta.

Asimismo, un elevado porcentaje de los productores mexicanos no son propietarios del equipo de trabajo y aún cuando éste sea de propiedad común (Sociedades Cooperativas) rara vez se destina un porcentaje de las ganancias para su mantenimiento. El pescador ocasional $o$ aquel que se ve sujeto a condiciones para tener acceso al equipo, puede coadyuvar involuntariamente a la depreciación del producto, para perjuicio del sector organizado.

Debe considerarse que tradicionalmente, México ha favorecido el desarrollo de sistemas industriales con políticas de mayor ga nancia a corto plazo pese a que requieren de mayor inversión, tienen un efecto negativo más evidente sobre el ambiente y están en riesgo de una rápida descapitalización por súbitas caídas del mercado o por colapso del recurso. En el caso de los pequeños productores, se ven obligados a asumir los riesgos y están en fuerte dependencia de agentes externos para la circulación de sus productos, lo que debilita su poder de negociación comparativamente con el sector privado. ${ }^{23}$ Por lo anterior y aunque es reiterativo mencionarlo, se hace necesaria una revisión de la política pesquera mexicana para ésta no concluya con la identificación que promueva permanentemente el consumo de diversos productos de la pesca. 
Asimismo, es importante considerar el caso de Els Alfacs, que aunque tampoco es representativo de la pesca ribereña en España, donde se presentan casos semejantes a los de Mazatlán y Bahía Bufadero, 24 muestra que a pesar de que los medios de producción no son colectivos, la organización social a otros niveles garantiza un adecuado nivel de vida a sus agremiados.

Particularmente, es necesario resaltar que existe control sobre el número de embarcaciones que operan en la zona, así como una administración del esfuerzo para regular la producción y los precios de venta. Es también importante la infraestructura que se ha generado con la colaboración de los socios, lo que les garantiza mantenimiento permanente, capacitación, bienestar social y especialmente, ser sujetos de crédito.

En ese sentido, sería recomendable que tanto a nivel local $\mathrm{Ma}$ zatlán y Bahía Bufadero) como regional, se organizaran los productores a fin de contar con calendarios ad hoc que les permitan actministrar su capacidad productiva, optimizar el uso de equipo y artes de pesca y al mismo tiempo, garantizar adecuados precios de mercado al mantener una oferta permanente. Esto daría lugar a un mejor aprovechamiento de la captura y a un abaratamiento de los costos de producción al contarse con mercado para la mayoría de las especies capturadas.

De igual manera, se considera que es urgente ubicar en sitios estra tégicos a las minúsculas, pero numerosas agrupaciones de pescadores, especialmente en lo que concierne a Michoacán, que podrían funcionar como centros de acopio, distribución y de ordenación de la producción, evitando así la saturación de mercados y proporcionando información al sector oficial, al ofrecerle elementos para una mejor administración de los recursos de la pesca. 


\section{Bibliografía}

INEGI (1993) M azatlán.Estado de Sinaloa,Cuaderno estadístico municipal. Gobierno del Estado de Sinaloa INEGI-Ayuntamiento de Mazatlán, $123 \mathrm{p}$.

(1996) El sector alimentario en M éxico, México, INEGI-CONAL,

$334 \mathrm{p}$.

Agüero, Max (1992), La pesca artesanal en América Latina:una visión pano rámica, Contributions for the study of artisanal fisheries in Latin America, ICLARM, no. 35, pp. 1-27

Camiñas, José Antonio (1988), Objetivos y estado de la investigación en la región surmediterránea española ( $M$ ar deA lborán), Memoria Jornadas sobre investigación de la pesca artesanal en el Mediterráneo español, C.S.C.I. Instituto de Ciencias del Mar de Barcelona, pp. 5768.

Delgado, Max (1986), Ecología de diatomeas costeras, relación plancton- ben tos, tesis doctoral, Universidad de Barcelona, 261 p.

Farrugio Henri, P. Oliver y F. Biagi (1993), An overview of thehistory, knowledge, recent and future research trends in Mediterranean fisheries, no. 57, pp. 105-119.

Flores Soria, Mario (1992), Edad y crecimiento en el Pargo Coconaco (Ho plopagrus gúntheri Gill.) en Playa norte,M azatlán,Sinaloa, M éxico, tesis de licenciatura, Facultad de Ciencias, UNAM.

García, M.M., J. Mendivil T., E. Franco O. y E. Vega A. (1985), Con tribución al conocimiento de la ictiofauna comercial capturada por la comuni dad pesquera "Playa norte" con un estudio de las relaciones biométricas de Ho plopagrus ü, ntheri Gill,M azatlán, Memoria del servicio social univer- 
sitario, Escuela Ciencias del Mar, Universidad Autónoma de Sinaloa, 127 pp.

Lleonart, Jordi (1988), La pesquería de Cataluña, informe final, Instituto de Ciencias del Mar de Barcelona, p. 220-235.

Macías Romo, Martha Elena y Asminda Felicidad Mota P. (1990), Algunos aspectos biológicos y pesqueros de la Sierra del Paćífico (Scomberomo rus sierra Jordan y Starks, 1895) en Bahía Bufadero, Michoacán y el Puerto de M azatlán,Sinaloa, tesis de licenciatura, México, Facultad de Ciencias, Universidad Nacional Autónoma de México, 154 p.

Madrid Vera, Juan (1990), Ecología de algunas especies de peces de importan cia comercial, tesis de maestría, Facultad de Ciencias, Universidad Nacional Autónoma de México, 179 p.

Madrid Vera, Juan, Pilar Sánchez y Arturo Ruiz Luna (en prensa), Diversity and abundance of a tropical fishery on the Pacific shelf of Michoacan, M exico, Est. Coast. Shelf Sci.

Millán, R.L. (1986), La pesca deSant Carles dela Ràpita en xifres/ cifras, Con fraria depescadors "Verge del Carme", Sant Carles de la Ràpita, 212 p.

Orbe, Araceli (1989), Los recursos pesqueros de la costa de Michoacán, Informe de labores, México, CRIP-Patzcuaro, 1986-1988. Instituto Nacional de Pesca, pp. 99-105.

Panayotou, Teodore (1982), M anagement concepts for small-scale fisheries: economic and social aspects, Roma, faO Fish. Tech. pap. 228, 53 p.

(1994), Ecología, medio ambiente y desarrollo, Debate crecimiento versus conservación, México, Gernika, 217 p.

Pielou, E.C. (1975) , Ecological diversity, New York, John Wiley \& Sons, $165 \mathrm{p}$. 
Ruiz Luna,Arturo (1983), Contribución al conocimiento de los peces mari nos de importancia comercial en Bahía Bufadero, Michoacán, M éxico, tesis de licenciatura, México, Universidad Nacional Autónoma de México, $137 \mathrm{p}$.

(1989), Descripción de la pesca y análisis de la variabilidad tempo ral de las capturas en la bahía Els Alfacs (Detta del Ebro), agosto de 1982 a julio de 1984, tesis de doctorado, Barcelona, Universidad de Barcelona, $335 \mathrm{p}$.

(1991), Fuentes de variación de la captura obtenida por la flota de artes menores en la bahía Els Alfacs, España, México, Instituto de Ciencias del Mar y Limnol, Universidad Nacional Autónoma de México, no. 18, pp. 89-98

Sardà, Francesc y Paloma Martín (1986), Les pesqueries a Catalunya: evo lució en Els últims decennis. In: L'oceanografia. Recursos pesquers de la mar ca talana, Quaderns díecologia aplicada, II. Barcelona, p. 91-112.

Semarnap ( Secretaría del Medio Ambiente Recursos naturales y Pesca) (1996), Programa de pesca y acuacultura 1995-2000, México. 